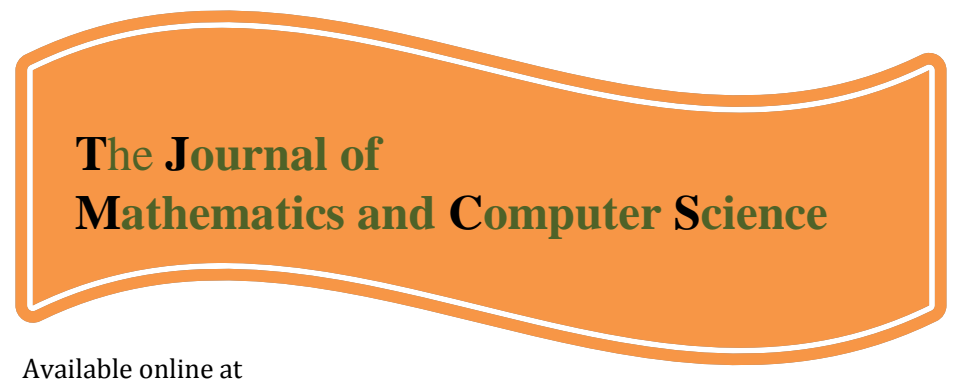

\title{
http://www.TIMCS.com
}

The Journal of Mathematics and Computer Science Vol .5 No.4 (2012) 313 - 319

\section{Extension of Pontryagin maximum principle and its Economical Applications}

\author{
Alireza Fakharzadeh \\ Department of Mathematics, Faculty of basic Sciences, Shiraz University of Technology,Shiraz, Iran \\ a_fakharzadeh@sutech.ac.ir \\ Somayeh Sharif \\ Department of Mathematics, Faculty of basic Sciences, Shiraz University of Technology, Shiraz, Iran \\ s.sharif@sutech.ac.ir
}

\author{
Karim Eslamloueyan \\ Department of Economics, Shiraz University, Shiraz, Iran \\ keslamlo@rose.shirazu.ac.ir
}

Received: February 2012, Revised: November 2012

Online Publication: December 2012

\begin{abstract}
First, an extension of Pontryagin Maximum Principle in Infinite-Horizon, which was presented by Aseev and Kryazhimiskii, is explained. Since this method is applicable in optimal economical growth problems, for the first time several problems such as consumption and investment are solved. Moreover, for implementing Aseev and Kryazhimiskii 's method on Iranian economy, Luis Serven model is introduced. Then it is calibrated on Iranian economy during the years 1385-1415. By applying the described method, the optimal consumption and investment for maximizing the social welfare are demonstrated. Also the sensitivity analysis is discussed.
\end{abstract}

Keywords: Economical Growth Models, Optimal Control, The Pontryagin Maximum Principle, Infinite Horizon

\section{Mathematics Subject Classification: Primary 91B62; Secondary 49J15.}

\section{Introduction.}

In general speaking, optimal control theory is made for solving dynamic optimization problems arise in technological control systems such as physics, robotics and aerospace (Garrard, 1977; Notsu et al., 2008). In several last decades, because of world population and restriction of energy sources and labor, this theory has very noted. Infinite-horizon optimal control problems emerge in many fields of economics, specially in optimal economic growth problems (EGP)(Aseev, 2008). Among the applications of optimal EGP, one may emphasize on optimal exploitation of both 
renewable and non renewable resources, optimal exploitation of a fish population, pollution control and optimal capital accumulation (Seierstad et al., 1987). Even there are various numerical or analytical approaches for solving optimal control problems, but one of the most important approaches in order to solving finite optimal control problems is still Pontryagin maximum principle (PMP) which was initiated by Pontryagin and his colleagues in 1962. However, his proposed method analytically investigates problems, but its approach cannot provide any information about the behavior of the adjoint variable and transversality conditions at infinite horizon. Designing the optimal EGP for infinite time leads to indicate the different extensions of PMP in infinite-horizon that we can point below cases (Aseev et al., 2008; 2007; 2004).

Halkin illustrated that taking the limits of transversality conditions could not be a valid extension in infinite-horizon (Halkin, 1974). Accordingly, methods were developed which were formed from PMP and another argument for obtaining transversality conditions. Under some concavity conditions of state and goal functions, Benveniste and Sheinkman extended the transversality conditions to infinite-horizon in 1982(Benveniste and Sheinkman, 1982). In 2001 Kamihigashi proved that the assumptions of unboundedness and homogeneity of goal function are sufficient for holding transversality conditions in infinite-horizon (Kamihigashi, 2001). After that under the assumptions that goal function is twice differentiable and the optimal pair is an interior point of a set in $\left(R^{n}\right)^{2}$, Long and Shimomura proposed the transversality conditions in 2003(Long and Shimomura, 2003). Then, Aseev and Kryazhimskii in 2007 establish a finite approximation approach for getting PMP in infinite-horizon, specially in optimal economic growth problems (Aseev and Kryazhimskii, 2007).

Since, optimal control theory has many applications in optimal economic growth models for infinite horizon, the main aim of this paper is to apply and fit the method advised by Aseev and Kryazhimiskii for the first time to these kind of problems (Aseev and Kryazhimiskiy, 2008). Moreover, calibrating the Luis Serven model (Serven, 1995), which is an optimal economic growth model, on Iranian economy is another important aims. In this manner, first, we express the proposed method by Aseev and Kryazhimskii and then, we show some of its applications on economical problems. Afterwards, we introduce the Luis Serven growth model which is contained all of this method properties. Then the required parameters are estimated by econometrics model. At the end, Luis Serven model is calibrated on Iranian economy.

\section{Aseev and Kryazhimiskii extension of PMP}

Following Aseev and Kryazhimskii (2008), Consider the optimal control problem (P):

$$
\begin{aligned}
& \operatorname{Max} J(x(t) \mu(t))=\int_{0}^{\infty} e^{-\rho t} g(x(t), u(t)), \\
& \dot{x}(t)=f(x(t), u(t)), \quad u(t) \in U, \quad(P) \\
& x(0)=x_{0} .
\end{aligned}
$$

Where $x \in R^{n}$ and $u \in R^{m}$ are respectively the state and control vectors; and $\mathrm{U}$ is a nonempty compact subset of $R^{m}$. Assume that there is an open set $G \in R^{n}$ such that $x_{0} \in G$ and $\rho \geq 0$ is a discount parameter. In addition, the functions $f: G \times U \rightarrow R^{n}$ and $g: G \times U \rightarrow R$ are supposed to be continuous and differentiable. Since problem (P) has many applications in economics (Arrow et al., 1970; Barro et al., 1995; Sethi et al., 2000), the below conditions is considered which are prevented the growth of the gradient of the instantaneous utility function $\mathrm{g}$ and the growth of admissible trajectories of the state system. These conditions are named (A1)-(A6) and introduced as below

(A1) There exist $C_{0} \geq 0$ such that

$$
\langle x, f(x, u)\rangle \leq C_{0}\left(1+\|x\|^{2}\right), x \in G, u \in U .
$$

(A2) For any $x \in G$, the set

$$
Q(x)=\left\{\left(z^{0}, z\right) \in R^{n+1}: z^{0} \leq g(x, u), z=f(x, u), u \in U\right\}
$$

is convex. 
(A3) There exist positive functions $\mu$ and $\omega$ on $[0, \infty)$ such that $\mu(t)$ and $\omega(t) \rightarrow+0$ as $t \rightarrow \infty$, and also the following inequalities holds for any admissible pair $(x, u)$ :

$$
\begin{aligned}
& e^{-\rho t} \max _{u \in U}\|g(x(t), u(t))\| \leq \mu(t), \forall t \geq 0, \\
& \int_{T}^{\infty} e^{-\rho t} \max _{u \in U}\|g(x(t), u(t))\| \leq \omega(t), \forall T \geq 0 .
\end{aligned}
$$

The existence of an optimal admissible pair of problem (P) is ensured by the conditions (A1)-(A3) (Aseev and Kryazhimiskiy, 2007; Balder, 1983).

(A4) There exist nonnegative constant $\mathrm{k}$ and $\mathrm{r}$ such that

$$
\left\|\frac{\partial g(x(t), u(t))}{\partial x}\right\| \leq k\left(1+\|x\|^{r}\right), x \in G, u \in U .
$$

(A5) There exist $C_{1}, C_{2}, C_{3} \geq 0$ and $\lambda \in R$ such that the following inequalities are satisfied for any admissible pair $(x, u)$ :

$$
\|x(t)\| \leq C_{1}+C_{2} e^{\lambda t},\left\|Y_{(x, u)}(t)\right\| \leq C_{3} e^{\lambda t}, \forall t \geq 0 .
$$

Note that $Y_{(x, u)}(t)$ is signified the normalized fundamental matrix of linear differential system

$$
\dot{y}(t)=\frac{\partial f(x(t), u(t))}{\partial x} y(t) .
$$

(A6) Conditions (A4),(A5) and the inequality $\rho(r+1)>\lambda$ are satisfied. The later condition is well-known as a dominating discount condition.

\subsection{Optimality Conditions}

Before introducing the optimality conditions for (P), reviewing the following definitions are necessary. The normal-form Hamiltonian function $H: G \times[0, \infty) \times R^{n} \rightarrow R$ is defined by $H(x, t, \psi)=\operatorname{Sup}_{u \in U} h(x, t, u, \psi)$ where $h(x, t, u, \psi)=\langle f(x, u), \psi\rangle+e^{-\rho t} g(x, u)$, and $\psi$ is an adjoint variable corresponding to $\left(x^{*}, u^{*}\right)$, if it is any solution of the following normal-form adjoint system on $[0, \infty)$ :

$$
\dot{\psi}(t)=-\left[\frac{\partial f\left(x^{*}(t), u^{*}(t)\right)}{\partial x}\right]^{*} \psi(t)-e^{-\rho t} \frac{\partial g\left(x^{*}(t), u^{*}(t)\right)}{\partial x} .
$$

An admissible pair $\left(x^{*}, u^{*}\right)$ and its adjoint variable $\psi$, satisfies the normal-form core relations of the PMP, if the maximum condition $h\left(x^{*}(t), t, u^{*}(t), \psi(t)\right) \stackrel{\text { a.e. }}{=} H\left(x^{*}(t), t, \psi(t)\right)$ holds on $[0, \infty)$. The optimality conditions of (P) are stated in the following theorems which can be founded in (Aseev and Kryazhimiskii, 2008; 2007) and their proofs are presented in (Aseev and Kryazhimiskii, 2004).

Theorem 2.1 Suppose that $\left(x^{*}, u^{*}\right)$ be an optimal admissible pair in problem (P) and conditions (A1)-(A3) hold. Then there exists an adjoint variable $\psi^{*}$ corresponding to $\left(x^{*}, u^{*}\right)$ which satisfies the normal-form core relations of Pontryagin maximum principle.

Theorem 2.2 Let $\left(x^{*}, u^{*}\right)$ be an optimal admissible pair of (P) and conditions (A1)-(A6) be satisfied. Then there exists an adjoint variable $\psi^{*}$ corresponding to $\left(x^{*}, u^{*}\right)$ such that the following conditions are satisfied:

(i) $\left(x^{*}, u^{*}\right)$, together with $\psi^{*}$, satisfies the normal-form core relations of the PMP on $[0, \infty)$.

(ii) for any $t \geq 0$, the stationary condition $H\left(x^{*}(t), t, \psi^{*}(t)\right)=\rho \int_{t}^{\infty} e^{-\rho s} g\left(x^{*}(s), u^{*}(s)\right) d s$ holds.

(iii) for every $t \geq 0, \quad I^{*}(t)=\int_{t}^{\infty} e^{-\rho s}\left[Z^{*}(s)\right]^{-1} \frac{\partial g\left(x^{*}(s), u^{*}(s)\right)}{\partial x} d s$ where $Z^{*}(t)=Z_{\left(x^{*}, u^{*}\right)}$, converges absolutely and $\psi^{*}(t)=Z^{*}(t) I^{*}(t)$. 


\subsection{The Economical Application.}

Based on the above discussions, we point to the some EGP which is solved by Aseev and Kryazhimiskii's method. To show the way of applying the mentioned optimality conditions in Theorems 2.1 and 2.2 for EGP, in this section we focus on solving some examples. By the proposed method, we solve some examples, which were figured out by Optimality Catching up Criterion(Seierstad and Sydsaeter, 1987). Note that comparing the obtained results indicated that even the both methods had the same analytical results, but the computations of the Aseev and Kryazhimiskii method is less. Now, we describe these examples.

Example1: Consider the following Optimal Control Problem:

$$
\begin{gathered}
\operatorname{Max} J(x(t) \mu(t))=\int_{0}^{\infty} \frac{1}{1-v} u(t)^{1-v} e^{-\rho t} d t, \\
\text { S to }: \quad \begin{array}{c}
\dot{K}=b K-u, \quad(P 2) \\
K(0)=K_{0}, \quad \lim _{x \rightarrow \infty} K(t) \geq 0 .
\end{array}
\end{gathered}
$$

where $v \in(0,1), b, K_{0}>0$ and $b-\rho<b v$. Assume that $\mathrm{K}$ and $\mathrm{u}$ represent the capital and consumption per worker, respectively. $\rho$ is a discount rate parameter. We consider $\frac{1}{1-v} u(t)^{1-v}$ and objective function as an utility function and the social welfare, respectively. Moreover, we suppose that $u \in U=\left[0, u_{\max }\right]$ and $\mathrm{K}$, the capital stock is finite and bounded. Thus, this problem is an EGP which is solvable by the forenamed optimality conditions. By some effort the conditions (A1)-(A6) are satisfied. So, we are ready to solve this problem by relying on theorem 2.2. First, we define the normal-form Hamiltonian function $H=\operatorname{Sup}_{u \in U} h$ where

$$
h=\frac{u(t)^{1-v}}{1-v} e^{-\rho t}+\psi(b K-u(t)) .
$$

Note that $\psi=c e^{-b t}$, is an adjoint variable of the normal form adjoint system $(2.1)$ on $[0, \infty)(c$ is a constant). According to (i) in Theorem 2.2, the optimal control is $u^{*}(t)=e^{\frac{-\rho t}{v}} \psi^{\frac{-1}{v}}=c^{\frac{-1}{v}} e^{\frac{(b-\rho) t}{v}}$. Then, $K^{*}(t)$ is obtained such that the condition (ii) is satisfied. So, $K^{*}(t)=\left(\frac{-v c^{\frac{-1}{v}}}{-b v+b-\rho}\right) e^{\frac{(b-\rho) t}{v}}$. Considering $K(0)=K_{0}$, it implies $K^{*}=K_{0} e^{\frac{(b-\rho) t}{v}}$. Also, we remind that ${ }^{*} \psi$ can be compute by using condition (iii). In this manner, the normalized fundamental matrix of adjoint system $\dot{Z}(t)=-b Z(t)$ is $Z^{*}(t)=e^{-b \rho}$. Since, $I^{*}(t)=0$ and $\psi^{*}(t)=0$.

Example2: Consider the below optimal control problem(Seierstad and Sydsaeter, 1987 page 247):

$$
\begin{gathered}
\operatorname{Max} \int_{0}^{\infty}(x(t)-u(t)) e^{-t} d t, \\
\dot{x}(t)=(u(t)-1) x(t), \quad(P 3) \\
x(0)=x_{0}>0, \quad x_{0} \neq 1,2 \quad, u \in[0,1], \\
x(\infty) \text { free. }
\end{gathered}
$$

Easily, in a similar way as stated in problem (P2), we can show the establishment of conditions (A1)-(A3). So, by relying on Theorem 2.1, we can find the optimal control and trajectory. In this manner, we define the normal Hamilton Pontryagin function as $h(t, x(t), u(t), \psi(t))=(x(t)-u(t)) e^{-t}+\psi(t)(u(t)-1) x(t)$ where $\psi$ is a solution of the following system on $[0, \infty)$

$$
\dot{\psi}(t)=-\frac{\partial h}{\partial x}=-e^{-t}+\psi(t)(1-u(t))
$$


According to (i), the maximum condition stated that $u^{*}$ maximize $h$ respect to $u$ or for any $t \geq 0$, $u^{*}$ maximizes

$$
\left(\psi(t) x(t)-e^{-t}\right) u(t) .
$$

Hence, $u^{*}$ is dependent on the sign of $d(t)=\psi(t) x(t)-e^{-t}$. So from (2.3) it follows that if $d(t) \geq 0$, then $u^{*}(t)=1$ and if $d(t)<0$ then $u^{*}(t)=0$ first, we assume that $d(t) \geq 0$, then $u^{*}(t)=1$ and the solution of (2.2) is $\psi(t)=e^{-t}$. Substituting $u^{*}(t)=1$ in state equation and using its initial condition, we get that $x^{*}(t)=x_{0} \quad$ Second, we assume that $d(t)<0$. So in a similar way as above we find. $x^{*}(t)=x_{0} e^{-t}$.

\section{The implementation of Luis Serven model for Iranian economy}

In this section, to focus on the simulation between Luis Serven model and problem (P) and moreover satisfying the assumptions such as the existence of infinite horizon and the homogenization of production functions, investment technology and consumption index, we introduce this model. Then, calibrate it on Iranian economy during the years 1385-1415. Afterwards, by using the method of Aseev and Kryazhimiskii was appointed the optimal consumption and investment for maximizing the social welfare.

Luis Serven model (Serven, 1995) was made by considering the effect of real exchange rate on Ramesy model. The aim of this model is to show the effect of capital goods imports with subject to adjustment costs on macroeconomic policies and external shocks. The Luis Serven model is briefed as follows:

$$
\begin{aligned}
& \operatorname{Max} \int_{0}^{\infty} \frac{C(t)^{1-\theta}}{1-\theta} e^{-\beta t} d t, \\
& \dot{K}(t)=I(t), \\
& \dot{A}(t)=\frac{1}{e}\left[Y(K)-P_{C}(e) C-P_{K}(e)\left(I+\frac{\phi I^{2}}{2 K}\right)\right]+r^{*} A, \\
& \lim _{t \rightarrow \infty} e^{-r^{*} t} A=0, K(0)=K_{0}, A(0)=A_{0} .
\end{aligned}
$$

where $C(),. I($.$) and K($.$) respectively are the real consumption, investment and capital stock. In$ addition to, the production technology is expressed by $Y(K)$ (with $\dot{Y}>0$ and $\ddot{Y}<0$ ) which in this paper is considered the

Cobb-Douglas function. Suppose further that $A$ denotes the agent's net foreign assets and the right hand side of $\dot{A}(t)$ in (3.1) denotes the agent's current account surplus. Also $e$ defines the real exchange rate (Serven, 1995).

\subsection{Calibration of the model.}

We calibrate the Luis Serven model on Iranian economy with the following parameter values which is determined exogenously and by the econometrics or the previous studies. Parameters of our calibration are mainly taken from previous studies. According to the reported estimations, we set the technology coefficient, $a$, and the elasticity of capital production, $b$, as $a=9$ and $b=0.62$ (Mahmoodzadeh et al., 1386). The discount rate, $\beta$, is set 0.01 (Din Mohammadi, 1387). Regarding the statistical sources of economic time series database, the labor growth rate, $g l$, is set to 0.037 . We choose the inverse of elasticity of intertemporal substitution, $\theta=0.66$ (Favero, 2005). The marginal installation cost, $\phi$, is about 0.05 according to the statistical sources of Iranian Ministry of Industries and Mines. Based on the statistical sources of IMF (International Monetary Fund), the world interest rate, $r^{*}$, is 0.04 . The value of capital deprecation rate, $\delta$, is cited 0.037 (Din Mohammadi, 1387). The remaining parameter values of calibration are estimated by 
econometrics. We remind that, $P_{C}(e)$ the consumption price index expresses the cost of one unit of real consumption in terms of the domestic goods. Furthermore, $P_{K}(e)$, the investment price index represents the cost of one unit of real investment in terms of domestic goods. Note that $P_{C}(e)$ and $P_{K}(e)$ are concave and strictly increasing functions (Serven, 1995). So,one could consider the estimable equation $P_{C}(e)=\alpha_{1} e+\beta_{1} e^{2}, P_{K}(e)=\alpha_{2} e+\beta_{2} e^{2}$ which have these properties. For these estimations, the data variables of $P_{C}(e), P_{K}(e)$ and $e$ is obtained from statistical sources during the years 1385-1415. Then by using Eviews5 software, the ordinary least square method and unit root test, the stationary of variables are investigated. Thus, we have

$$
P_{C}(e)=1.24 e+0.29 e^{2}, P_{K}(e)=1.31 e+0.31 e^{2} .
$$

Now, the parameters are ready to solve problem (3.1)by Aseev and Kryazhimiskii's method.

\section{Solution of economic growth problem}

By using the calibrated parameters of section 3.1, we try to solve the problem (3.1) with Aseev and Kryazhimiskii extension of PMP's method. Easily we can show the conditions (A1)-(A6) are satisfied, so theorem 2.2 can be used to obtain the solution. Applying theorem 2.2 to problem (3.1) transfers it to a system of nonlinear differential equation which is solvable with MATLAB2007 software; in this manner, the problem is solved and the obtained optimal trajectories (capital and net foreign assets) and optimal controls (consumption and investment) are shown in Figure1. In addition, the maximum social welfare is attained as 10828.2258 .
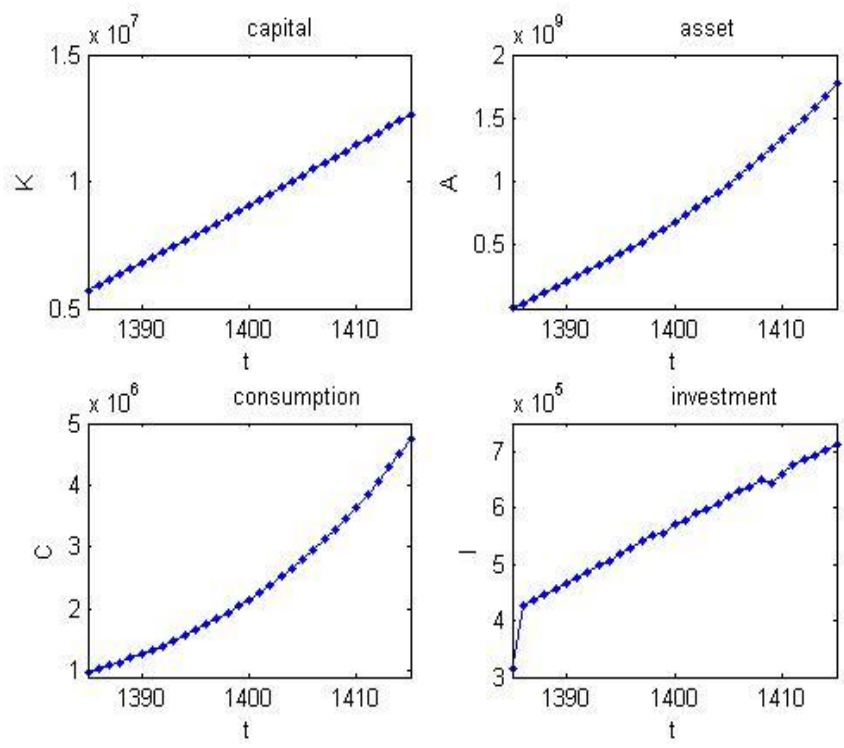

Figure1. Optimal controls and trajectories

Now, we investigate the sensitivity analysis at parameters of discount rate and real exchange rate. Moreover, their effects on social welfare are appointed.

By increasing the value of discount rate and resolving the problem by the described method, these values decrease the social welfare. Because, this accession in objective function means that the consumption at the present time is important than the future. So, by decreasing the consumption and the direct relationship between consumption and welfare, the welfare is reduced.

Accession of the real exchange rate (depreciation) causes that the capital stock and the social welfare has negatively related. This fact is adapted with the reality. Since, depreciation means that getting foreign goods expensive than domestic goods and foreign goods effect on generating capital goods in country. So, it causes to increase the cost of capital goods, accordingly, the optimal capital stock is reduced.

\section{Conclusion}

The aim of this paper was to emboss the capability of Aseev and Kryazhimiskii 's method for solving optimal economical growth problems in infinite horizon. In this manner, for the first time 
several optimal economical growth problems solved analytically by this method. The obtained results showed the capability, validity and accuracy of this method. Moreover, For representing the native application of the described method. Luis Serven model was studied in Iranian Economy. Then by satisfying conditions A1-A6, theorem 2.2 was used for solving the calibrated Luis Serven 's model. Furthermore, the optimal control and state paths were extracted during the years 13851415. The calibrated maximum social welfare is $1.08282258 \times 10^{4}$. Afterwards, the sensitivity of model for real exchange rate and discount rate were discussed. Sensitivity analysis results show that the accession of discount rate reduces the social welfare and the accession of the real exchange rate increase the social welfare.

\section{References.}

[1] A. Amini and M. Neshat, Estimated time series of the investment in Iran's economy during the period 1338-1381, J. Planning and Budget, 90 (1384).

[2] K.J. Arrow and M. Kruz, Public Investment, the Rate of Return, and Optimal Fiscal Policy , J. Hopkins University Press, Baltimore, MD, (1970).

[3] S. M. Aseev, Infinite-Horizon Optimal Control with Applications in Growth Theory, Proceeding of the Stoklove Institute of Mathematics, 262 (2008), 10-25.

[4] S. M. Aseev and A. V. Kryazhimiskiy, Shadow prices in infinite-horizon optimal control problems with dominating discounts, Applied Mathematics and Computation, 204 (2008), 519-531.

[5] S. M. Aseev and A. V. Kryazhimiskii, The Pontryagin maximum principle and optimal economic growth problems, Proceeding of the Steklove Institute of Mathematics, 257 (2007), 1-255.

[6] S. M. Aseev and A. V. Kryazhimiskiy, The Pontryagin maximum principle and transversality conditions for a class of optimal control problems with infinite time horizons, Control Optim, 43 (2004), 1094-1119.

[7] S. M. Aseev and A. V. Kryazhimiskii, The Pontryagin maximum principle for an optimal control problem with a functional specified by an improper integral, Dokl. Math., 69 (2004), 89-91.

[8] E. J. Balder, An existence result for optimal economic growth problems , J. Math. Anal. Appl., 95 (1983), 195-213.

[9] R. J. Barro and X. Sala-i-Martin, Economic Growth, McGraw-Hill, New York, (1995).

[10] L. M. Benveniste and J. A. Sheinkman, Duality theory for dynamic optimization models of economics: The continuous time case, Journal of Economic Theory, 27 (1982), 1-19.

[11] M. Din Mohammadi, provide a model for optimal allocation of natural gas resources in Iran, PhD Dissertation, Esfahan University, (1387).

[12] C. A. Favero, Consumption, Wealth, the Elasticity of Intertemporal Substitution and Long-Run Stock Market Returns, IGIER, Bocconi University and CEPR, (2005).

[13] W. L. Garrard and J. M. Jordan, Design of nonlinear automatic flight control systems, Automatica, 13 (1977), 497-505.

[14] H. Halkin, Necessary Conditions for optimal control problems with Infinite Horizons, Econometria, 42 (1974), 267-272.

[15] T. Kamihigashi, Necessity of transversality conditions for infinite horizon problems, Econometrica, 69 (2001), 995-1012.

[16] N. V. Long and K. Shimomoura, A note on transversality conditions, RIEB Discussion Paper Series 144, Koba University, (2003).

[17] M. Mahmoodzadeh and F. Asadi, Effects of information and communication technologies on labor productivity growth in the Iranian economy, Journal of Business, 43 (1386).

[18] T. Notsu, M. Konishi and J. Imai, Optimal water cooling control for plate rolling, Int. J. Innov. Comput. Inform. Control, 4 (2008), 3169-3181.

[19] A. Seierstad and K. Sydsaeter, Optimal Control Theory with Economic Applications, NorthHolland, Amsterdam, (1987).

[20] L. Serven, Capital goods imports, the real exchange rate, and the current account, International Economics, 39 (1995), 79-101.

[21] S. Sethi and G.I. Thompson, Optimal Control Theory, Applications to Management Sciences and Economics, Kluwer, Dordrecht, (2000). 